\title{
FUNCTIONS WHOSE DERIVATIVES TAKE VALUES IN A HALF-PLANE
}

\author{
FERNANDO GRAY AND STEPHAN RUSCHEWEYH
}

(Communicated by Irwin Kra)

\begin{abstract}
We derive sharp upper and lower bounds for $\left|z f^{\prime}(z) / f(z)\right|$ where $f \in \mathscr{R}$, i.e. $f$ analytic in $\mathbf{D}$ with $f(0)=0, f^{\prime}(0)=1$ and $\operatorname{Re} e^{i \alpha} f^{\prime}(z)>0$ in $\mathbf{D}$ for a certain $\alpha=\alpha(f) \in \mathbf{R}$. The extremal function is $k(z)=-z-2 \log (1-z)$. This result improves an earlier one of D. K. Thomas.
\end{abstract}

Let $\mathscr{R}_{0}$ denote the class of analytic functions $f$ in the unit disk $\mathbf{D}$ with $f(0)=0$, $f^{\prime}(0)=1$, and $\operatorname{Re} f^{\prime}(z)>0$ in D. In a recent paper D. K. Thomas [4] proved that for $f \in \mathscr{R}_{0}$ one has

$$
\left|\frac{z f^{\prime}(z)}{f(z)}\right| \leq \frac{-K}{(1-|z|) \log (1-|z|)}, \quad z \in \mathbf{D},
$$

where $K$ is an absolute but unknown constant. The example $k \in \mathscr{R}_{0}$ with $k(z)=$ $-z-2 \log (1-z)$ shows that the bound in (1) is of the right order. In this note we obtain the sharp version of (1), including also the lower bound and prove that $k$ is extremal in both cases. In fact, our theorem holds for the larger class $\mathscr{R}$ where the condition $\operatorname{Re} f^{\prime}(z)>0$ is replaced by $\operatorname{Re} e^{i \alpha} f^{\prime}(z)>0$ in $\mathbf{D}$ for some $\alpha=\alpha(f) \in \mathbf{R}$.

THEOREM. For $f \in \mathscr{R}$ and $|z| \leq r<1$ we have

$$
\frac{1-r}{1+r} \frac{r}{-r+2 \log (1+r)} \leq\left|\frac{z f^{\prime}(z)}{f(z)}\right| \leq \frac{1+r}{1-r} \frac{-r}{r+2 \log (1-r)} .
$$

Both sides of the estimate are sharp for $k$ at $z=-r$ and $z=r$, respectively.

Frequently relations like (2) indicate that there is a subordination lurking behind; in this case it could be

$$
z f^{\prime}(z) / f(z)<z k^{\prime}(z) / k(z)
$$

This, however, is not true: using a recent result [3, Lemma 2] one can show that $k$ is starlike univalent in $\mathbf{D}$. Then (3) would imply that $f$ is starlike univalent if $f \in \mathscr{R}$. Since this is not the case we have a contradiction to (3).

The difference between $\mathscr{R}$ and $\mathscr{R}_{0}$, as far as the theory for solving extremal problems of the type under consideration is concerned, is the known structure of possible extremal functions. According to [1] the only candidates for extremizing $\left|z f^{\prime}(z) / f(z)\right|$ over $\mathscr{R}_{0}$ are the functions $f$ with

$$
f^{\prime}(z)=\lambda \frac{1+x z}{1-x z}+(1-\lambda) \frac{1+y z}{1-y z}, \quad|x|=|y|=1,0 \leq \lambda \leq 1 .
$$

Received by the editors June 30, 1987 and, in revised form, September 2, 1987.

1980 Mathematics Subject Classification (1985 Revision). Primary 30C45.

Research supported by the Fondo Nacional de Desarollo Científico y Tecnológico (Grant 249/87) and the Universidad F. Santa María (Grant 87.12.06). 
It is clear that maximizing the functional is not easy even for this restricted class of functions. On the other hand, it follows from the Duality Principle [2, Theorems $1.1,1.6$, Corollary 1.1] that the functional over $\mathscr{R}$ takes its maximum for one of the functions $f$ with

$$
f^{\prime}(z)=(1+x z) /(1+y z), \quad|x|=|y| \leq 1 .
$$

With this information the problem becomes manageable (not completely trivial, though). And we are lucky enough that the extremal function for $\mathscr{R}$ (namely $k$ ) lies in $\mathscr{R}_{0}$, hence solving the problem for $\mathscr{R}_{0}$ as well. We shall require a lemma.

LEMmA. Let $F(z):=1+z / \log (1-z)$ and $G(z):=(1-F(z)) /(1-z)$. Then $F$ and $G$ have nonnegative Taylor coefficients about $z=0$ and, in particular, for $|z| \leq r<1$,

$$
\begin{gathered}
|F(z)| \leq F(r) \leq \lim _{t \rightarrow 1} F(t)=1, \quad\left|F^{\prime}(z)\right| \leq F^{\prime}(r), \\
|G(z)| \leq G(r) .
\end{gathered}
$$
$H$,

$$
(1-z)\left(z H^{\prime}(z)-H(z)\right)=H^{2}(z) .
$$

If $H(z)=\sum_{n=0}^{\infty} a_{n} z^{n}$ then (7) becomes

$$
\sum_{n=0}^{\infty}\left[(n-1) a_{n}-(n-2) a_{n-1}\right] z^{n}=\sum_{n=0}^{\infty} \sum_{j=0}^{n} a_{j} a_{n-j} z^{n}
$$

where $a_{-1}:=0$. Comparing coefficients in (8) we obtain for $n \geq 1$,

$$
(n-1) a_{n}=(n-2) a_{n-1}+\sum_{j=0}^{n} a_{j} a_{n-j},
$$

and since $a_{0}=-1$ we may rewrite this as

$$
\begin{gathered}
0=-a_{0}+2 a_{0} a_{1}, \quad n=1, \\
(n+1) a_{n}=(n-2) a_{n-1}+\sum_{j=1}^{n-1} a_{j} a_{n-j}, \quad n>1 .
\end{gathered}
$$

Relation (9) gives $a_{1}=1 / 2>0$ and an induction using (10) proves $a_{n}>0, n \geq 2$. The inequalities (5) follow.

Writing $G(z)=\sum_{n=0}^{\infty} b_{n} z^{n}$ we deduce

$$
b_{n}=1-\sum_{j=1}^{n} a_{j}=1-\lim _{t \rightarrow 1} \sum_{j=1}^{n} a_{j} t^{j} \geq 1-\lim _{t \rightarrow 1} F(t)=0,
$$

which completes the proof.

We now turn to the proof of the Theorem. Clearly the set of possible extremal functions (4) can be further reduced to

$$
f^{\prime}(z)=(1+x z) /(1-z), \quad|x|=1 .
$$


We start with the lower bound and write

$$
\frac{f(z)}{z f^{\prime}(z)}=\int_{0}^{1} \frac{f^{\prime}(t z)}{f^{\prime}(z)} d t=\int_{0}^{1} \frac{1+x t z}{1-t z} \frac{1-z}{1+x z} d t .
$$

Now, for $0 \leq t \leq 1$, we have

$$
\frac{1+t|z|}{1+|z|} \leq\left|\frac{1+t z}{1+z}\right| \leq \frac{1-t|z|}{1-|z|}, \quad z \in \mathbf{D}
$$

Hence

$$
\left|\frac{1+x t z}{1+x z} \frac{1-z}{1-t z}\right| \leq \frac{1-t|z|}{1-|z|} \frac{1+|z|}{1+t|z|}
$$

and

$$
\left|\frac{f(z)}{z f^{\prime}(z)}\right| \leq \frac{1+r}{1-r} \int_{0}^{1} \frac{1-t r}{1+t r} d t=\frac{k(-r)}{-r k^{\prime}(-r)},
$$

the desired result. For the upper bound we write with (11) and the notation of the Lemma

$$
\begin{aligned}
\left|\frac{z f^{\prime}(z)}{f(z)}\right| & =\left|\frac{1+x z}{1-z} \frac{1}{x+(1+x) \log (1-z) / z}\right| \\
& =\left|\frac{1+x z}{1+x F(z)} \frac{1-F(z)}{1-z}\right| .
\end{aligned}
$$

Maximizing with respect to $|x|=1$ leads to

$$
\left|\frac{1+x z}{1+x F(z)}\right| \leq \frac{|z-F(z)|+|1-F(z) \bar{z}|}{1-|F(z)|^{2}},
$$

and thus

$$
\begin{aligned}
\left|\frac{z f^{\prime}(z)}{f(z)}\right| & \leq|G(z)| \frac{|z-F(z)|+|1-F(z) \bar{z}|}{1-|F(z)|^{2}} \\
& =\frac{1}{1-|F(z)|^{2}}\left\{r\left|G(z)\left(1-\frac{F(z)}{z}\right)\right|+|G(z)|\left|1-r^{2}+r^{2}\left(1-\frac{F(z)}{z}\right)\right|\right\} \\
& \leq \frac{1}{1-|F(z)|^{2}}\left\{r(1+r)\left|G(z)\left(1-\frac{F(z)}{z}\right)\right|+\left(1-r^{2}\right)|G(z)|\right\} \\
& =\frac{1+r}{1-|F(z)|^{2}}\left\{r\left|F^{\prime}(z)\right|+(1-r)|G(z)|\right\} .
\end{aligned}
$$

In the last step we used $F^{\prime}(z)=G(z)(1-F(z) / z)$. As a consequence of the Lemma we deduce that the last expression is maximal for $z=r$ and an evaluation in that point gives the right-hand side of (2) (that this is true for $|z|<r$ as well is trivial).

ADDENDUM. After having completed this manuscript we became aware of the paper On functions whose derivative has positive real part by $\mathrm{R}$. $\mathrm{R}$. London (to appear), in which a slightly stronger version of the right-hand inequality in (2) has been established which, however, holds only for functions $f \in \mathscr{R}_{0}$ :

$$
\frac{\left|f^{\prime}(z)\right|}{\operatorname{Re} f(z) / z} \leq \frac{1+r}{1-r} \frac{-r}{r+2 \log (1-r)}, \quad|z| \leq r .
$$

Since London's proof of (12) is fairly complicated we take the opportunity to show that our method gives (12) with almost no calculations: let $f \in \mathscr{R}_{0}$. Then obviously 
$\operatorname{Re}(f(z) / z)>0$ in $\mathbf{D}$ and, according to [1, Theorem 1.b] we have for every $\varepsilon$ with $|\varepsilon|=1$ and $|z|=r$ a $|z(\varepsilon)|=r$ such that

$$
\frac{\operatorname{Re} \varepsilon f^{\prime}(z)}{\operatorname{Re} f(z) / z}=\frac{\operatorname{Re} k^{\prime}(z(\varepsilon))}{\operatorname{Re} k(z(\varepsilon)) / z(\varepsilon)} \leq \frac{\left|k^{\prime}(z(\varepsilon))\right|}{\operatorname{Re} k(z(\varepsilon)) / z(\varepsilon)} \leq \max _{|z|=r} \frac{\left|k^{\prime}(z)\right|}{\operatorname{Re} k(z) / z}
$$

and thus, using $\varepsilon=\left|f^{\prime}(z)\right| / f^{\prime}(z)$,

$$
\frac{\left|f^{\prime}(z)\right|}{\operatorname{Re} f(z) / z} \leq \max _{|z|=r} \frac{\left|k^{\prime}(z)\right|}{\operatorname{Re} k(z) / z}
$$

Now, with the notation and the results of the Lemma and its proof we get for $|z| \leq r$

$$
\begin{aligned}
\frac{\left|k^{\prime}(z)\right|}{\operatorname{Re} k(z) / z} & =\left|\frac{1+z}{1-z}\right| \frac{1}{-1-2 \operatorname{Re} \log (1-z) / z} \\
& =\left|\frac{1+z}{1-z}\right| \frac{1}{|\log (1-z) / z|^{2}-|1+\log (1-z) / z|^{2}} \\
& =\frac{1}{1-|F(z)|^{2}}\left|\frac{1+z}{1-z}\left(\frac{z}{\log (1-z)}\right)^{2}\right| \\
& =\frac{1}{1-|F(z)|^{2}}\left|\frac{1+z}{1-z} H^{2}(z)\right| \\
& \stackrel{(7)}{=} \frac{|1+z|\left|z H^{\prime}(z)-H(z)\right|}{1-|F(z)|^{2}} \\
& \leq \frac{(1+r)\left(r H^{\prime}(r)-H(r)\right)}{1-F(r)^{2}}=\frac{r k^{\prime}(r)}{k(r)} .
\end{aligned}
$$

Here we made use of the fact that $z H^{\prime}-H$ as well as $F(z)$ has positive coefficients. This proves (12).

\section{REFERENCES}

1. S. Ruscheweyh, Nichtlineare Extremalprobleme für holomorphe Stieltjesintegrale, Math. Z. 142 (1975), 19-23.

2. _ Convolutions in geometric function theory, Séminaire de Mathématiques Supérieures, 83, Presses de l'Univ. Montréal, 1982.

3. ___ Coefficient conditions for starlike functions, Glasgow Math. J. 29 (1987), 141-142.

4. D. K. Thomas, On functions whose derivative has positive real part, Proc. Amer. Math. Soc. 98 (1986), 68-70.

Departmento de Matemática, Universidad Técnica Federico Santa María, VALPARAÍSO, CHILE 